\title{
Research on multi-sensor information fusion and intelligent optimization algorithm and related topics of mobile robots
}

\author{
Yuan Guo ${ }^{1}$, Xiaoyan Fang ${ }^{2 *}$, Zhenbiao Dong ${ }^{1}$ and Honglin Mi ${ }^{1}$
}

\author{
${ }^{*}$ Correspondence: \\ gongpingshizhe@126.com \\ ${ }^{2}$ School of Foreign \\ Languages, Shanghai \\ Institute of Technology, \\ Shanghai 201418, China \\ Full list of author information \\ is available at the end of the \\ article
}

\begin{abstract}
Research on mobile robots began in the late 1960s. Mobile robots are a typical autonomous intelligent system and a hot spot in the high-tech field. They are the intersection of multiple technical disciplines such as computer artificial intelligence, robotics, control theory and electronic technology. The product not only has potentially very attractive application value and commercial value, but the research on it is also a challenge to intelligent technology. The development of mobile robots provides excellent research for various intelligent technologies and solutions. This dissertation aims to study the research of multi-sensor information fusion and intelligent optimization methods and the methods of applying them to mobile robot related technologies, and in-depth study of the construction of mobile robot maps from the perspective of multi-sensor information fusion. And, in order to achieve this function, combined with autonomous exploration and other related theories and algorithms, combined with the Robot Operating System (ROS). This paper proposes the area equalization method, equalization method, fuzzy neural network and other methods to promote the realization of related technologies. At the same time, this paper conducts simulation research based on the SLAM comprehensive experiment of the JNPF-4WD square mobile robot. On this basis, the high precision and high reliability of robot positioning are further realized. The experimental results in this paper show that the maximum error of the $X$-axis and $Y$-axis, FastSLAM algorithm is smaller than EKF algorithm, and the improved FASTSALM algorithm error is further reduced compared with the original FastSLAM algorithm, the value is less than 0.1 .
\end{abstract}

Keywords: Multi-sensor, Information fusion, Algorithm research, Intelligent optimization, Mobile robot, Route plan

\section{Introduction}

\subsection{Background}

With the advancement of science and technology and the continuous development of society, the birth of robots is an important product of the development of science and technology, and the research on robots has become an unstoppable scientific boom, which has been highly valued by various industries, especially industry and academia. As the world's most advanced automation system, intelligent robots are highly valued

(c) The Author(s), 2021. Open Access This article is licensed under a Creative Commons Attribution 4.0 International License, which permits use, sharing, adaptation, distribution and reproduction in any medium or format, as long as you give appropriate credit to the original author(s) and the source, provide a link to the Creative Commons licence, and indicate if changes were made. The images or other third party material in this article are included in the article's Creative Commons licence, unless indicated otherwise in a credit line to the material. If material is not included in the article's Creative Commons licence and your intended use is not permitted by statutory regulation or exceeds the permitted use, you will need to obtain permission directly from the copyright holder. To view a copy of this licence, visit http:// creativecommons.org/licenses/by/4.0/. 
by countries all over the world. How to use technology to realize the industrialization of mobile robots and obtain corresponding results is the key issue of current research. At the same time, the working environment of mobile robots is not only applied to the traditional single indoor environment, but also gradually applied to many complex outdoor environments, even the outer space environment. However, autonomous mobile robots involve extensive and complex technologies. Unlike traditional industrial robot control systems, most of the corresponding control technologies for mobile robots remain in the theoretical stage and have not been verified in actual practical stages, so mobile robots are not as good as people. It appears in people's lives in large numbers as expected. In the past ten years, with the rapid development of artificial intelligence and robotics, various intelligent decision support systems, expert systems, learning algorithms, fuzzy algorithms, neural network algorithms, intelligent planning algorithms, etc., have been applied to various intelligent robot systems.

\subsection{Significance}

With the increasing application of sensor technology, this technology has been gradually applied to intelligent mobile robots. Robot technology has become a very promising industry that has a significant impact on the country's economic development and people's lives. With the continuous development of mobile robot technology, a variety of sensors are gradually applicable to various intelligent robot fields. Therefore, multisensor information fusion technology is widely used in mobile robot research. The mobile robot perception system, as well as the human perception system, are responsible for obtaining information about the robot's internal and external environment (humans), so that the mobile robot can automatically supply power to the corresponding work instructions and functional content. Because information fusion technology can improve the speed, accuracy and stability of the robot mobile system, the multi-sensor mobile robot system can be coordinated and controlled so that each sensor uses the corresponding information processing technology: to meet the overall requirements. Therefore, the research on multi-sensor information fusion technology has been in an active historical stage. In addition, SLAM research also includes sensor technology, mathematical statistics (or computer technology), computer technology, artificial intelligence and other industries. In-depth research will be able to find a traceable solution to a genius mobile robot. Improve the level of robotics research in my country. At the national strategic level, a large number of 863 projects have been implemented, including the research and development of high-tech mobile robot technology, and large universities are also fully developing mobile robots.

\subsection{Related work}

With the development of intelligence and information society, the research of mobile robots has attracted the attention of many experts and scholars at home and abroad. The robot obtains its own state information and external environment information through sensors and relies on this information to realize autonomous movement and complete certain tasks. Since it is difficult for a single sensor to obtain all the information required by the system, the information fusion of multiple sensors becomes the key to the robot's autonomous decision-making. In the single-parameter diagnosis of complex systems, 
$\mathrm{JiB}$ aims at incomplete information and uncertainty and proposes a multi-sensor information fusion error diagnosis method based on neural BP network and D-S evidence theory. This method is applied to the fault diagnosis of a certain type of rocket launcher hydraulic drive servo system, and the fault location and diagnosis of the main components of the hydraulic drive servo system are realized [1]. Yi proposed a new multisensor information fusion fault diagnosis method based on BP neural network and D-S evidence theory for the problem of incomplete information and uncertainty in the single parameter diagnosis of complex systems. This method is applied to the fault diagnosis of a certain type of rocket launcher hydraulic drive servo system (HDSS), and the fault location and diagnosis of the main components of the hydraulic drive servo system are realized [2]. Omid has proposed several methods to extract various heuristics and high-level features from gait movement data to identify distinctive gait features and distinguish the target individual from other individuals. However, manual and handmade feature extraction is error-prone and subjective. In addition, the motion data collected from inertial sensors have a complex structure, and the separation between the manual feature extraction module and the predictive learning model may limit the generalization ability [3]. Although the scholars have made some achievements in related research, there are some problems with the viewpoints raised by the scholars, the experimental procedures and the methods used in the research process.

\subsection{Innovation}

The innovation of this article is (1) First of all, the innovation of the topic selection angle. This article is a new perspective from the perspective of topic selection. At present, there are not many researches that integrate the four of multi-sensor, information fusion, algorithm research, intelligent optimization and mobile robot. It is of exploratory significance. (2) The second is the innovation of research methods. This paper uses fuzzy logic and fuzzy neural network information fusion algorithm, area equalization method and other methods, and applies the algorithm to the research of mobile robots. (3) The other is the innovation of project practice. Multi-sensor information fusion technology has been applied to intelligent mobile robots, which has had a significant impact on the development of the national economy and people's lives.

\section{Multi-sensor information fusion algorithm}

\subsection{EM algorithm}

\subsubsection{Calculation of antecedents of fuzzy rules}

The basic principle of multi-sensor information fusion technology is like the process of the human brain's comprehensive information processing. Various sensors are processed for multi-level and multi-space information complementary and optimized combination processing, and finally a consistent interpretation of the observation environment is produced. In this process, it is necessary to make full use of multi-source data for reasonable control and use, and the ultimate goal of information fusion is to derive more useful information based on the separated observation information obtained by each sensor, through the multi-level and multi-faceted combination of information. This not only takes advantage of the cooperative operation of multiple sensors, but also comprehensively processes data from other information sources to improve the intelligence of 
the entire sensor system. Set the observation data set $K$ and the non-observation data set $X$ to form a new set $E$, and denote it as $E=(K, Z)$. It is customary to call the set $K$ a complete data set, and the observation data set $X$ as an incomplete data set. The essential idea of EM algorithm [4] is successive approximation and continuous optimization. Although the parameters of the selected model are unknown, by randomly selecting some parameters or setting initial parameters based on experience, the EM algorithm can start from the given initial parameters to obtain the optimal state corresponding to this set of parameters:

$$
\operatorname{probor}\left(\chi_{\mathrm{A}}(\kappa), \chi_{\mathrm{B}}(\kappa)\right)=\chi_{\mathrm{A}}(\kappa)+\chi_{\mathrm{B}}(\kappa)-\chi_{\mathrm{A}}(\kappa) \cdot \chi_{\mathrm{B}}(\kappa)
$$

Among them, $A, B$ is the fuzzy set on $\kappa$, and $\chi_{A}, \chi_{B}$ is the corresponding degree of membership.

\subsubsection{Use fuzzy rules to infer the subsequent parts of fuzzy rules}

After obtaining the antecedents of the fuzzy rules, it is necessary to use fuzzy implication calculations to derive the subsequent fuzzy rules according to the rule base. The fuzzy implication relation "-" means "if yes then yes". The commonly used fuzzy implication operators are:

$$
\begin{aligned}
& A \rightarrow \mathrm{B}=\chi_{\mathrm{A}}(\kappa) \cdot \chi_{\mathrm{B}}(\kappa) \\
& A \rightarrow \mathrm{B}=\max \left(\min \left(\chi_{\mathrm{A}}(\kappa), \chi_{\mathrm{B}}(\kappa)\right), 1-\chi_{\mathrm{A}}(\kappa)\right)
\end{aligned}
$$

In most cases, the system has multiple inputs and multiple outputs, its structure is complex, and the rules are closely related. The fuzzy rule base has the following form:

$$
W=\left\{R_{\mathrm{MIMO}}^{1}, R_{\mathrm{MIMO}}^{2}, \ldots R_{\mathrm{MIMO}}^{n}\right\}
$$

Due to the mutual independence between the output variables, the reasoning and judgment of the system will be replaced by the system.

\subsubsection{Maximum membership degree method}

The principle of maximum membership is the first principle of exhaustion, that is, all molecules in the object population can be classified. The second is the principle of exclusivity, that is, no molecule in the object population can belong to two or more categories at the same time. According to the reference, we give the recursive EM-FKF algorithm [5]. For the local filter i, its state equation can be described as:

$$
\chi_{D}\left(d_{0}\right) \geq \chi_{D}(d), \quad d \in D
$$

\subsubsection{Area balance method}

The area equalization method is also called the median method [6], which takes the value of the segmented straight line and the segmented axis of the area surrounded by the component function curve as the accurate output value, that is: 


$$
\int_{b}^{G_{n}} \chi_{\mathrm{A}}(H) \mathrm{d} z=\int_{G n}^{b} \chi_{\mathrm{A}}(H) \mathrm{d} z
$$

\subsubsection{Center of gravity method}

The center of gravity method obtains the update of the error state by maximizing the likelihood function [7]. This step and the fusion of the common state in the main filter are performed at the same time:

$$
z_{0}=\frac{\int_{a}^{b} z \chi_{D}(z) \mathrm{d} z}{\int_{a}^{b} \chi_{D}(z) \mathrm{d} z}
$$

\subsection{Fuzzy neural network}

A basic unit of the neural network is the neuron [8], which is equivalent to the closure of the human nervous system. Any neuron can perform a specific function and has a certain information processing capability, so it is also called a node.

\section{3 is the $n$ output signals of the neuron $j$, and 2 is the weight of the input signal. The artificial neuron is expressed in mathematical expressions as follows:}

$$
O_{J}=f\left(\sum_{i=1}^{n} W_{i j} X_{i}-T_{j}\right)
$$

From the above derivation process, we can get: to calculate $O$, you need to use the Kalman filter to calculate the conditional expectation of the public state and its variance. For the federated Kalman filter [9]. BP neural network has arbitrarily complex pattern classification capabilities and excellent multi-dimensional function mapping capabilities and solves the exclusive OR (XOR) and some other problems that simple perceptrons cannot solve. Structurally, the BP network has an input layer, a hidden layer and an output layer; in essence, the BP algorithm uses the square of the network error as the objective function and uses the gradient descent method to calculate the minimum value of the objective function. The network structure diagram is shown in Fig. 1.

If $N$ training samples are provided in the learning and training stage of the network [10], then in the feedforward calculation of the BP neural network, we can operate in this way. Information fusion, also known as data fusion, is a technology that comprehensively processes and optimizes the acquisition, presentation and internal relations of multiple types of information. The sensor information after fusion has the following characteristics: information redundancy, information complementarity, real-time information and low information acquisition. Randomly select samples 1 input and 2 output to provide the system for learning and training. The input of the $i$ th neuron of the layer can be written as: 


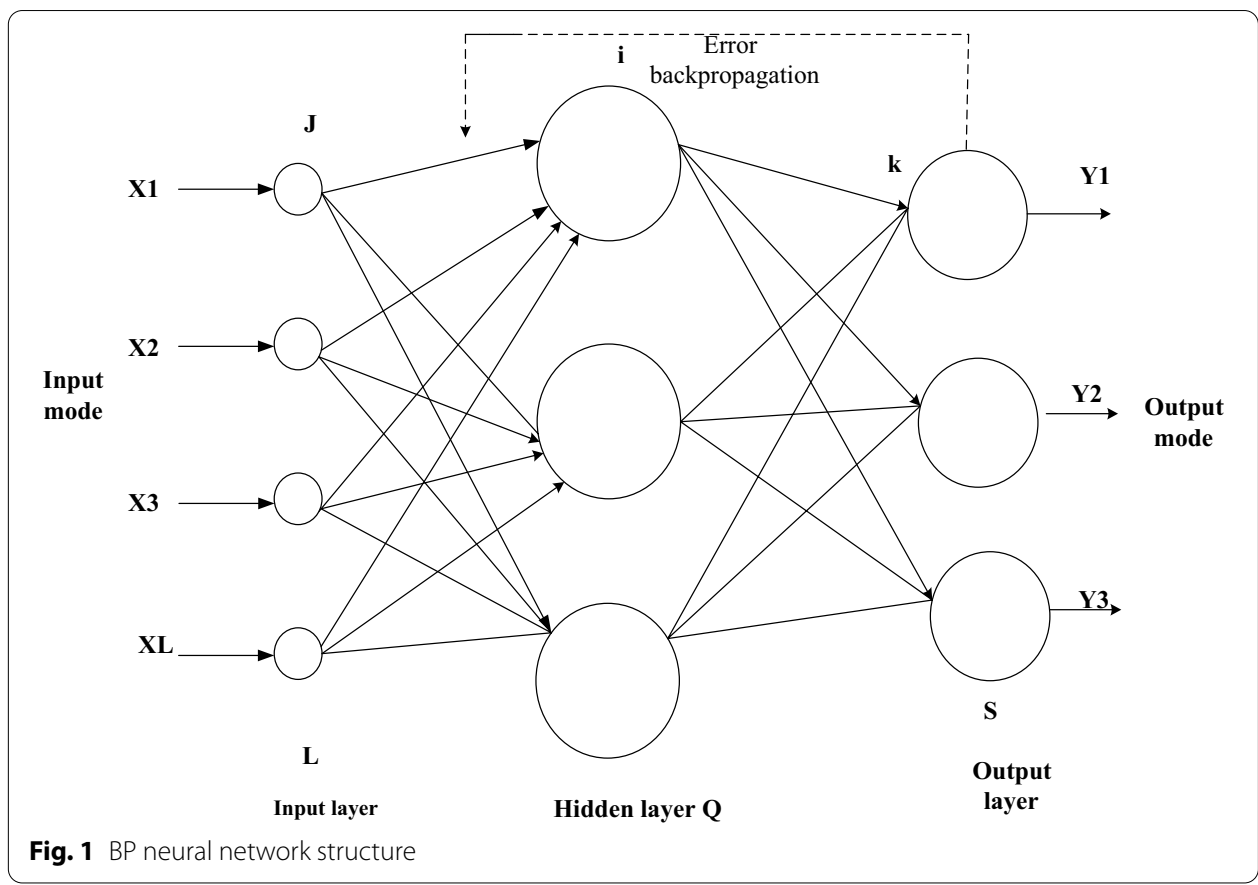

$$
\operatorname{met}_{i}^{1}=\sum_{j=1}^{l} w_{i j} o_{j}^{l}-\theta_{i}=\sum_{j=1}^{L} w_{i j} x_{j}^{1}-\theta_{i}
$$

\subsection{Fuzzy neural network}

Through calculation, we can conclude that the TS (Takagi Sugeno) fuzzy model is characterized by the fact that the consequent part of the fuzzy rule depends on the input and is a combination of input variables, while the latter part of the traditional fuzzy model consists of the fuzzy set of the output [11] composition. The predecessor records the position of the previous node of the node, and the successor records the position of the next node of the node. The algorithm formula of its model is as follows:

$$
y_{i}=p_{i 0}+p_{i 1} x_{1}+p_{i 2} x_{2}+\cdots+p_{i n} x_{n}
$$

Introducing the structure and learning ability of the neural network into the fuzzy model can form a model fuzzy system based on the neural network, which is called the fuzzy neural network. Its network structure is shown in Fig. 2.

It can be seen from Fig. 2 that the predecessor network and the postpart network are formed together to form a fuzzy network. The antecedent network is composed of four layers to generate the antecedent parameters of fuzzy rules [12]; the subsequent network is composed of three layers to generate fuzzy rule conclusions.

Set input quantitative 1 and the fuzzy language set of input variable 2 as:

$$
T\left(x_{i}\right)=\left\{W_{e 1}, W_{e 2}, \ldots, W_{e n}\right\}, e=1,2,3, \ldots, n
$$

The first layer is the input layer. Pass each input value $X=\left\{x_{1}, x_{2}, x_{3}, \ldots, x_{n}\right\}^{e}$ volunteer to the second layer. The second layer is the membership layer. The membership 


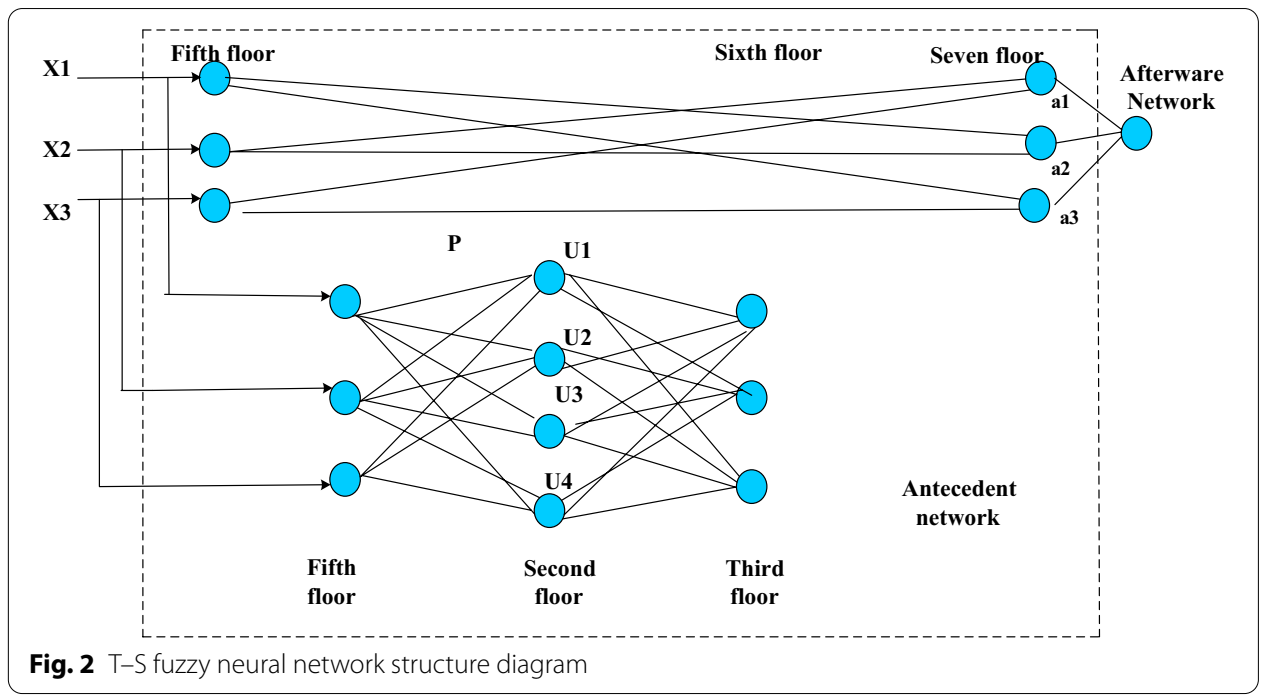

function $v_{i j}$ is to calculate that the input variables belong to each language variable set, namely:

$$
v_{i j}=v_{s c}\left(x_{n}\right)
$$

If the membership function adopts the normal function [13], then:

$$
v_{i j}=\exp \frac{\left(x_{i}-c_{i j}\right)^{2}}{\partial_{i j}^{2}}
$$

The third layer is the rule layer, which calculates the fitness of each rule. The input of the node of this layer is the membership value of a language variable of each input variable. Different language variables of the same input variable [14] cannot be used as the input of the same node, which is:

$$
s_{j}=\min \left\{\mu_{1 i}, \mu_{2 i}, \ldots, \mu_{n i}\right\}
$$

Or

$$
s_{j}=\mu_{1 i}, \mu_{2 i}, \ldots, \mu_{n i}
$$

\section{Experimental section: comprehensive experiments based on mobile robots}

\subsection{Simulation research}

This article is based on the MATLAB7.0 simulation platform. The sensor area is $50 \mathrm{~m} \times 50 \mathrm{~m}$, and the space field is $100 \mathrm{~m} \times 100 \mathrm{~m}$. In terms of shape, the small triangle represents the actual position of the robot, the circle represents the position of the anchor knot [15], the square represents the robot position estimated by the improved four-fold distance algorithm, and the diamond represents the estimated position of the robot using the four-side distance algorithm. The shape represents the estimated position of the robot using the three-side distance algorithm. The system parameters of the mobile robot are shown in Table 1. 
Table 1 System parameters of a single static positioning of a mobile robot

\begin{tabular}{lccc}
\hline Parameter & Algorithm & \\
\cline { 2 - 4 } & Three margin & Four margins & $\begin{array}{c}\text { Improve the } \\
\text { four margins }\end{array}$ \\
\hline Positioning error $(\mathrm{m})$ & 41.5663 & 17.63 & 3.256 \\
Computing time $(\mathrm{s})$ & $0.6523 \%$ & 0.18563 & 0.19563 \\
\hline
\end{tabular}

From Table 1, the data show that the algorithm not only has high reliability, effectively avoids the phenomenon of information flooding, but also improves the positioning accuracy of the robot without increasing the communication cost, the third position algorithm and the fourth distance algorithm are compared to the improved four-sided distance range algorithm [16] However, the position failure is small and short calculation time. The positioning error is shown in Fig. 3.

The average positioning error of the mobile robot is shown in Table 2.

In the case that the anchor nodes are all 10 and are randomly given, the comparison of the data in Table 2 shows that the improved four-side ranging positioning algorithm has small errors, stable algorithms and high reliability, and the number of anchor nodes changes [17] as shown in Fig. 4.

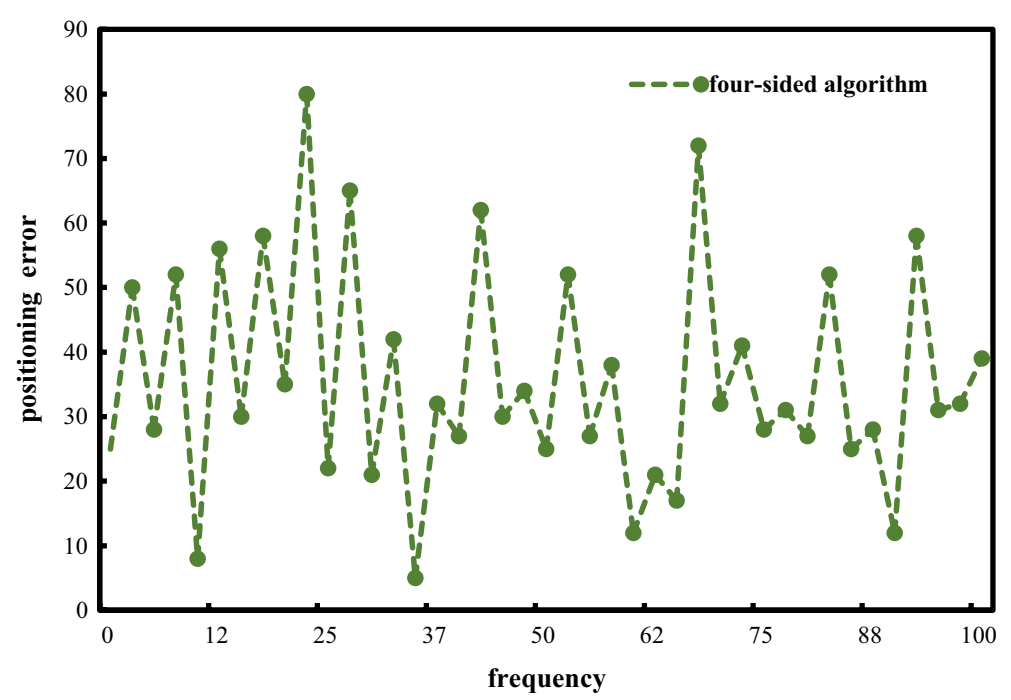

Fig. 3 Simulation diagram of the error of the trilateral ranging positioning algorithm

Table 2 Average positioning error of multiple static positioning of the mobile robot

\begin{tabular}{llcl}
\hline $\begin{array}{l}\text { Positioning error } \\
\text { Average value }\end{array}$ & Algorithm & \\
\cline { 2 - 4 } & Three margin & Four margins & $\begin{array}{l}\text { Improve the } \\
\text { four margins }\end{array}$ \\
\hline 10 times & 26.253 & 8.756 & 2.5698 \\
40 times & 29.563 & 14.526 & 2.369 \\
80 times & 45.639 & 17.856 & 3.7598 \\
\hline
\end{tabular}




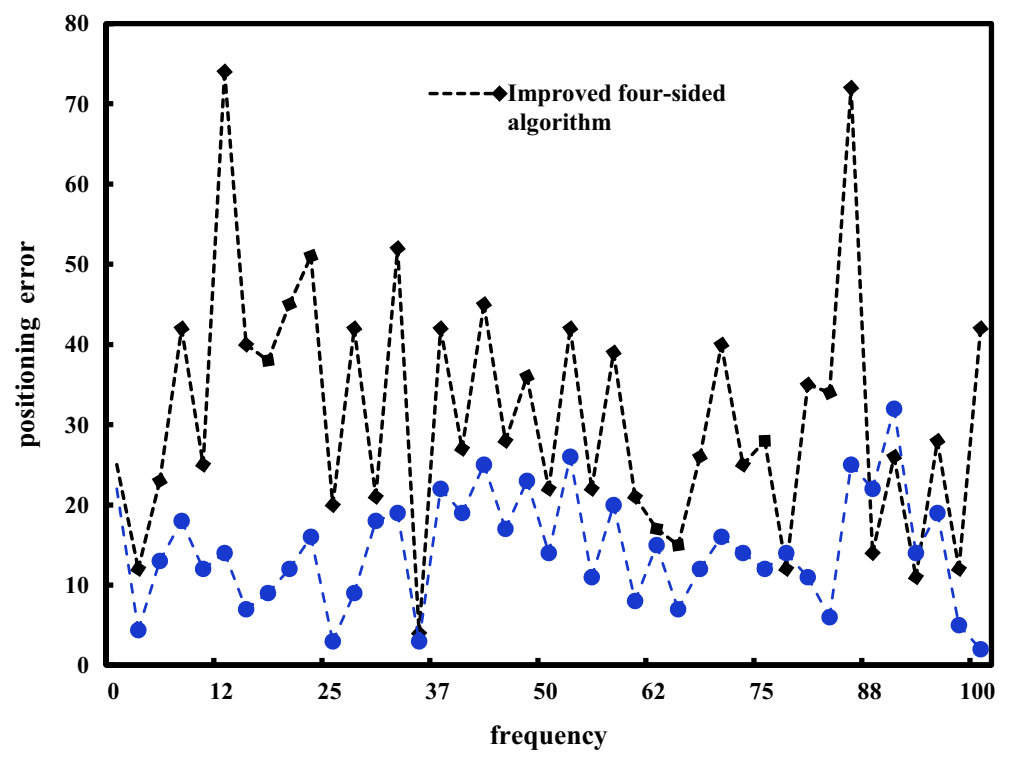

Fig. 4 Simulation diagram of two positioning algorithms when the number of anchor nodes changes

Figure 4 shows the error of the three-party positioning algorithm and the four-party positioning algorithm. From the four aspects of location allocation algorithm improvement, each time it is from 5 to 100 . Under the same hardware conditions, the stability of the algorithm is improved from four aspects. The position error is small and the accuracy is high. This paper presents an improved quadrilateral positioning algorithm. There is no need for iteration, with good speed and recommended weighted scarab positioning data. RSSI [18] completely controls the position of each node through the distance factor. The influence of positioning accuracy helps to avoid data phenomenon and data loss of St. Tor's algorithm. The anchoring 918 between the used distance and the strength of the signal is achieved by using the same accuracy of communication conditions. And the high reliability of the robot has been improved.

\subsection{System modeling of mobile robots}

The Simulink module provided by MATLAB software is an intuitive, convenient and efficient simulation tool. In the Simulink simulation environment, drag each module to the model window and connect according to the corresponding relationship to establish a simulation model of the system. The fuzzy controller of this obstacle avoidance system adopts the fuzzy logic controller module provided by Simulink tool [19], and is named Robot. Enter Robot1 = readfis ("Robot") command in the control window of MATLAB to establish a connection to the system. In the simulation environment, the robot's operating environment is first established, and the coordinate origins $(0,0)$ and $(10,10)$ are, respectively, used as the starting position and target position of the robot. Mobile roboter har mobilkonstruksjonen på hjul blitt mye brukt. Hovedårsaken er at sammenlignet med andre drivstrukturer er strukturen på hjulene enkel, handlingen er stabil og det er lettere å kontrollere, og kontrollmetoden er mer intuitiv. This means that all wheels moving on a plane must meet the constraints of moving around a common instantaneous 
center of rotation (ICR). Although all kinds of wheeled mobile robots have a variety of mobile mechanisms and forms, according to the different combinations of mobility and steering of mobile robots [20], mobile robots that move on a plane and meet rigid body constraints are divided into five types. Types of. The calculated input LD, FD, RD and Angle are used as the input of the fuzzy controller, and the real-time speed of the robot is output through the fuzzy inference mechanism. Connect the $m$ file with Simulink in real time, that is, the calculated output value of the $\mathrm{m}$ file is used as the input of the fuzzy controller in Simulink, and the output of the fuzzy controller is fed back to the $\mathrm{m}$ file. The connection diagram between the m file and Simulink [20] is shown in Fig. 5.

Combine the robot's kinematics theory with the actual external environment, continuously adjust the parameters of each module and simulate the collected environmental data.

\subsection{The basic model of multi-sensor information fusion}

The most critical part of model design is data integration, and the working model and the fusion model belong to the fusion algorithm data fusion model. The main functions of the database and the interaction process between the various parts of the data synchronization system describe the integration of the data. Structural model, data fusion, software describing the hardware components of the data fusion system and other related models are the external environment of the mathematical model.

\subsubsection{Functional model}

The most reliable information fusion function model is the Joint Committee of the Data Fusion Expert Group of the US Military Government Technical Committee. The model divides data fusion into three levels. The first level is the processing of one source or multiple sources, mainly digital processing [21], monitoring and correlation. The second level is the basis for evaluating the overall situation, a series of object evaluations and evaluations of interrelationships. The third level uses a target set on the system for confirmation and evaluation.

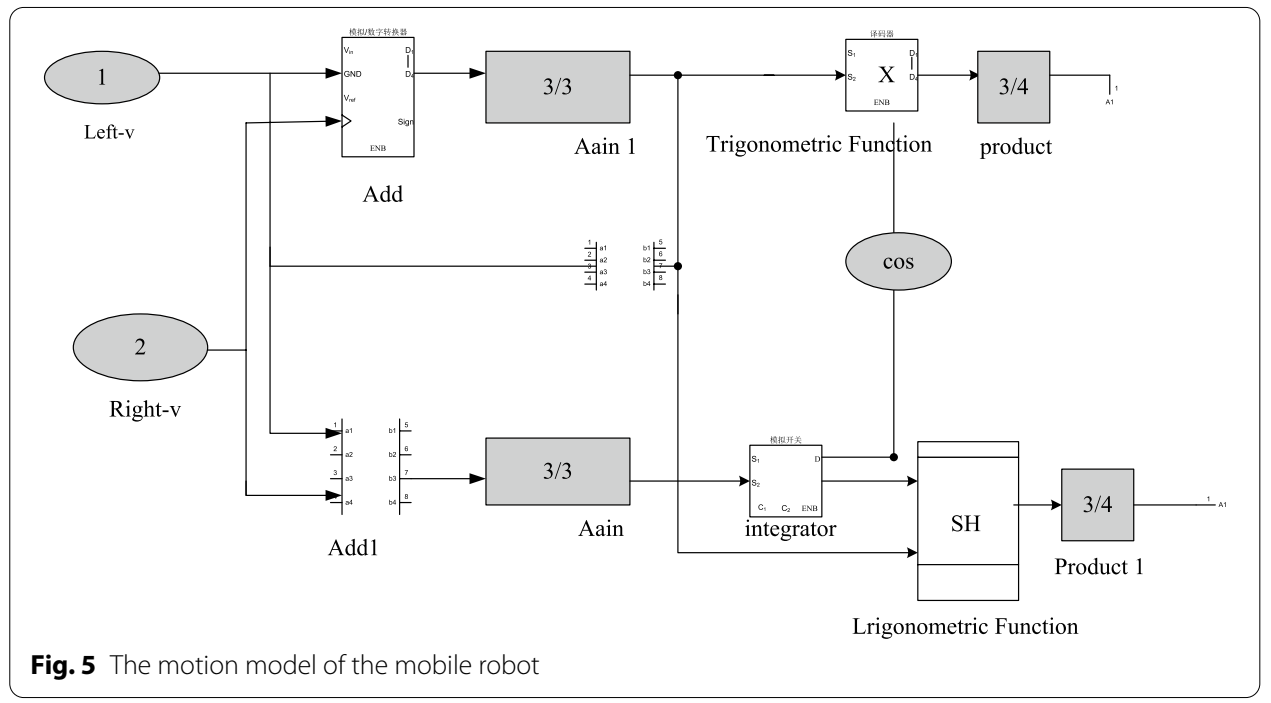




\subsubsection{Structural model}

There are many ways to classify the structure model of information fusion. The classification standard analyzes the data processing process and classifies the fusion structure, which is divided into data-level pixel-level synthesis [22], feature-level synthesis and crystal-level synthesis. Data level fusion is the lowest level of fusion, directly fusing sensor observation data, and then extracting features and making decisions based on the fusion results. The integration of the characteristic level belongs to the middle-level integration. First, each sensor deletes its own characteristic range [23], which may be edge, direction, speed, etc. The fusion center should complete the fusion process through extended characteristics. Generally, the derived feature information is a complete representation of the data information or sufficient statistical data elements. The fusion of decision-making is a high-level fusion. First, each sensor makes a decision based on its own data, and then the fusion center completes part of the fusion process in the decision-making process [24]. The advantages and disadvantages of the three levels of information fusion are shown in Table 3.

The fusion model of a system is usually composed of multiple fusion units at different levels, that is, multiple fusion units. On this basis, the system fusion unit is divided into four sub-units: combination, synthesis, fusion and association.

\subsubsection{Mathematical model}

Multi-sensor information fusion algorithm is an important part of multi-sensor information fusion. In recent years, the commonly used multi-sensor fusion methods can be divided into four categories: estimation methods, recognition methods, recognition methods, parameters and artificial intelligence methods.

\section{Discussion section}

\subsection{Improve the experimental analysis of extended Kalman}

In order to predict the pose of the mobile robot and obtain an accurate pose estimate, an error motion model is added to the measurement model with an odometer. Based on improved Kalman fusion block diagram, we can design the positioning flowchart as shown in Fig. 6.

In the MATLAB/SIMULINK software environment, the simulation experiment of the movement of the mobile robot is designed, and the positioning accuracy of the mobile robot is analyzed using the fusion idea of improved Kalman filter [25]. If the parameters of the sonar sensor are set to 1 : the linear velocity is $0.15 \mathrm{~m} / \mathrm{s}$, the angular velocity is the sampling time $T=0.1 \mathrm{~s}$, and the matching threshold is 3 . The corresponding simulation initial condition is 4 , the motion noise covariance matrix

Table 3 Comparison of the characteristics of the three integration levels

\begin{tabular}{|c|c|c|c|c|c|c|c|}
\hline $\begin{array}{l}\text { Integration } \\
\text { level }\end{array}$ & $\begin{array}{l}\text { Information } \\
\text { loss }\end{array}$ & $\begin{array}{l}\text { Information } \\
\text { loss }\end{array}$ & Precision & $\begin{array}{l}\text { Fault } \\
\text { tolerance }\end{array}$ & $\begin{array}{l}\text { Anti-dry } \\
\text { ability }\end{array}$ & $\begin{array}{l}\text { Calculation } \\
\text { amount }\end{array}$ & $\begin{array}{l}\text { Integration } \\
\text { level }\end{array}$ \\
\hline Data layer & Small & Poor & High & Poor & Poor & Big & Low \\
\hline Feature layer & Middle & Medium & Medium & Medium & Medium & Middle & Medium \\
\hline $\begin{array}{l}\text { Decision- } \\
\text { making layer }\end{array}$ & Big & Good & low & Excellent & Excellent & Small & High \\
\hline
\end{tabular}




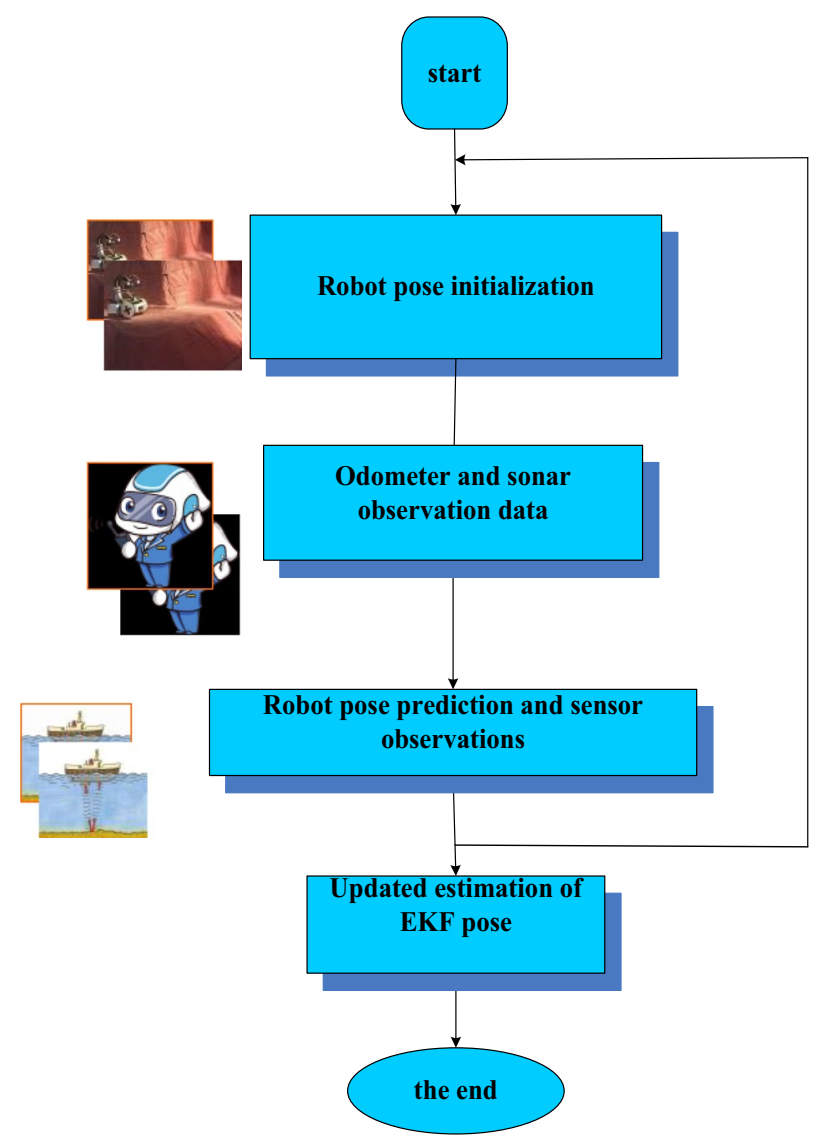

Fig. 6 Improved Kalman information fusion flow chart

is $R=\operatorname{diag}[0.1,0.1,0.1]$. Combining the previous positioning method, using the improved Kalman filter fusion idea of sentiment accuracy to write a simulation program, the simulation results obtained on MATLAB are shown in Figs. 7 and 8.

The simulation results in Fig. 7 show that the error of the mobile robot simulation positioning is reduced. Figure 8 shows the $X$-axis and $Y$-axis error analysis diagrams. From the figure, it can only be due to the Gaussian error model at the beginning. The resulting filter divergence problem, the error amount has basically approached to 0 after $15 \mathrm{~s}$, especially the $X$-axis error is smaller. Therefore, the sonar sensor used in this paper is used as an auxiliary sensor to correct the movement error of the mobile robot, which provides a certain guarantee for the final estimation of the actual physical position. The problem of sudden errors and error accumulation that a single sensor may bring in the pose estimation of mobile robots is discussed, and an improved Kalman algorithm that emphasizes accuracy is proposed to fuse mobile robot sonar sensor information to solve this problem. And by discussing the odometer's periodic correction error during the update process, as well as the matching between the sonar sensor's observation value and the predicted value, the relevant fusion model is established, so as to improve the positioning accuracy requirements, and then the fusion performance conduct analysis and research. For the source of the degradation 


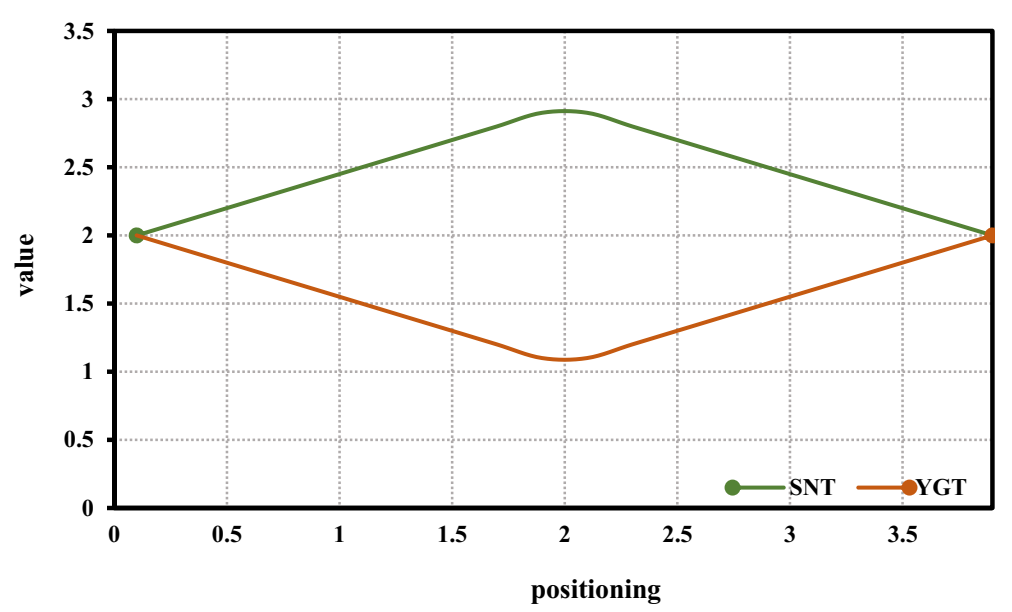

Fig. 7 Kalman positioning simulation of mobile robot

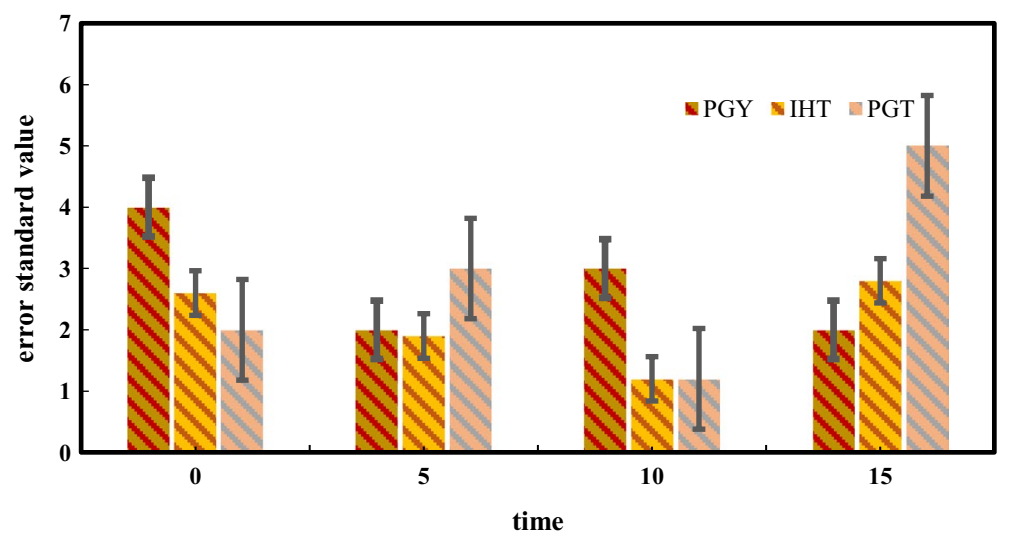

Fig. 8 Simulation error graph

of Kalman filter performance-inaccurate estimation of the observation noise, a series of optimization treatments have been carried out.

\subsection{Analysis of experimental results}

The experimental simulation environment is Windows10, Matlab2013, and the experimental instrument uses a notebook with Intel i5 processor and 8G memory. This experiment uses the open-source simulation platform of Professor Tim Bailey to conduct comparative experiments on EKFSLAM, FastSLAM and the improved IAPSO-FastSLAM, and then analyzes based on the experimental results. The simulation experiment constructed a $200 \mathrm{~m} \times 200 \mathrm{~m}$ ring map. Among them, there are 21 waypoints, and 65 landmark points are calibrated around the waypoints to imitate the landmark information observed by the binocular camera. Before the start of the experiment, set the robot to move from $(0,0)$. The speed of the movement is $5 \mathrm{~m} / \mathrm{s}$, the error of the movement is $0.5 \mathrm{~m} / \mathrm{s}$, the maximum steering angle of the movement is 40 degrees, and the maximum steering angle change rate is 25 degrees/s. The error 


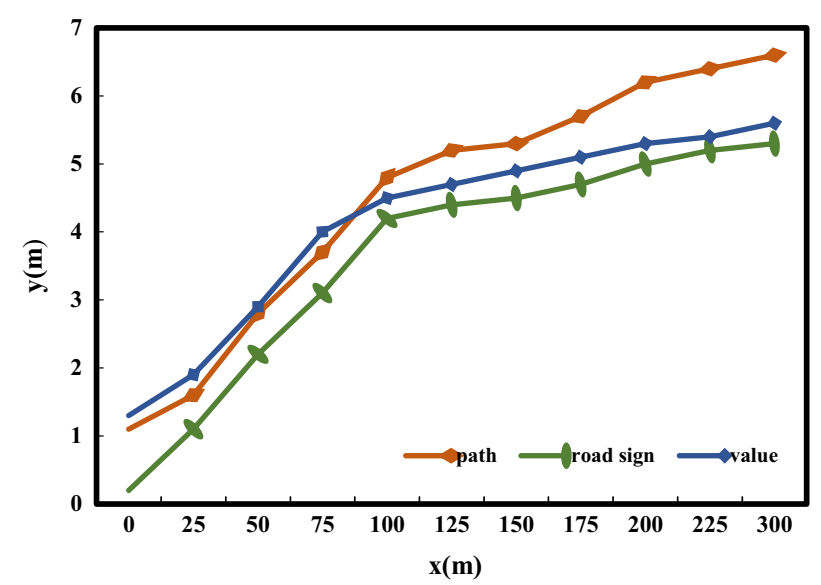

Fig. 9 Simulation map and landmarks

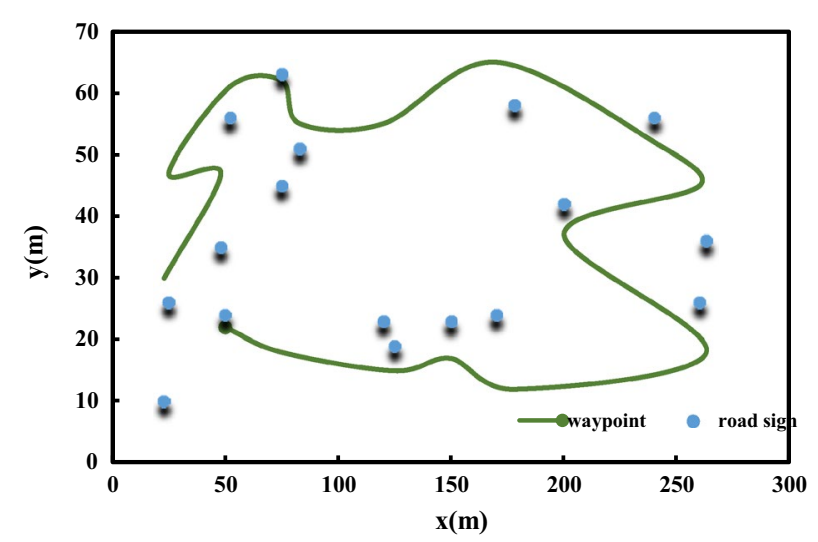

Fig. 10 EKFSLAM simulation results

is 4 degrees. The observed distance error is $0.2 \mathrm{~m}$, and the angle error is 1 degree. The covariance matrix of the movement process can be obtained as:

$$
Q=\left[\begin{array}{cc}
0.6^{2}, & 0 \\
0, & (3 * \pi, 180)
\end{array}\right]
$$

The covariance matrix of the observation process is:

$$
R=\left[\begin{array}{cc}
0.3^{2}, & 0 \\
0, & (1 * \pi / 180)^{2}
\end{array}\right]
$$

The average value of the robot's pose was recorded through simulation, and the number of particles in this experiment was 15 . The map results used in the simulation are shown in Figs. 9, 10 and 11.

From the above three figures, it can be seen that the simulation result of the EKFSLAM algorithm is the worst. The estimated motion trajectory and the real motion trajectory of the robot have a large error. The result of the improved FastSLAM 


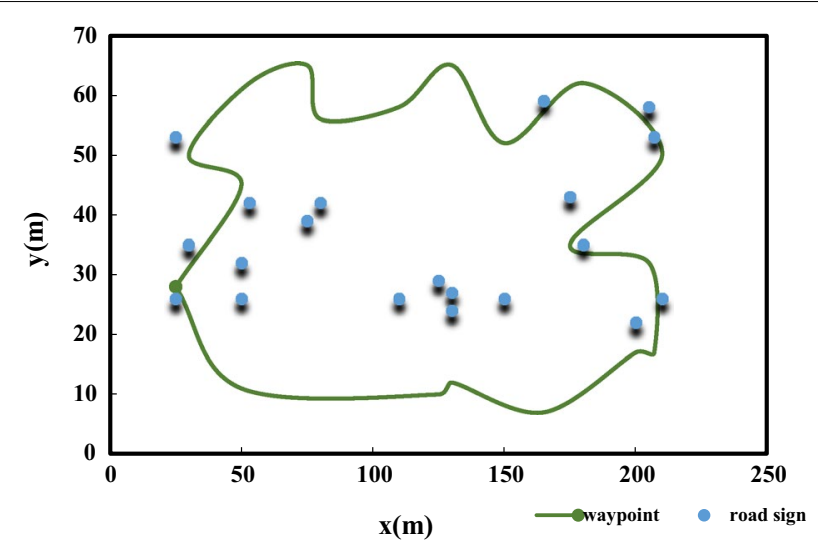

Fig. 11 IA-PSO-FastSLAM algorithm simulation results

Table 4 Error statistics

\begin{tabular}{lllll}
\hline Algorithm & Time/s & $\begin{array}{l}\text { X-axis maximum } \\
\text { error/m }\end{array}$ & $\begin{array}{l}\text { Y-axis maximum } \\
\text { error/m }\end{array}$ & $\begin{array}{l}\text { Maximum } \\
\text { angle error/ } \\
\text { rad }\end{array}$ \\
\hline EKFSLAM & 43.26 & 2.96 & 3.56 & 0.25 \\
FastSLAM & 116.25 & 1.36 & 2.36 & 0.036 \\
IA-PSO-PFSLAM & 126.36 & 1.04 & 1.93 & 0.056 \\
\hline
\end{tabular}

algorithm is better than the original FASTSALM algorithm, and the positioning is more accurate and more suitable. At the same time, it can be observed that the improved FastSLAM algorithm is obviously more accurate than the original FastSLAM algorithm in terms of the $X$-axis and $Y$-axis errors. Then use Table 4 to calculate the maximum error.

It can be seen from Table 4 that the maximum error of the $X$-axis and $Y$-axis, FastSLAM algorithm is smaller than the EKF algorithm, and the improved FASTSALM algorithm error is further reduced compared to the original FastSLAM algorithm. Through the statistical table, it can be concluded that optimizing the particle set through the fusion of immune algorithm and particle swarm algorithm can effectively bring the particles closer to the real position and make the distribution of particles closer to the true posterior probability of the robot. At the same time, it can be seen from the comparison of time that the FastSLAM algorithm runs longer than the EKFSLAM algorithm. Because FastSLAM is more complex, the EKFSLAM algorithm runs the shortest. The execution time of the improved FastSLAM algorithm is longer than that of the FastSLAM algorithm, because in order to get better results, the improved FastSLAM algorithm uses immune algorithms and particle swarm algorithms instead of direct resampling, which requires iterative convergence, resulting in increases in execution time, but it can still meet the real-time requirements of the SLAM algorithm. The algorithm used to integrate the IMU and the binocular camera to solve the SLAM problem is the FastSLAM algorithm, and it points out the shortcomings of the FastSLAM algorithm. Then, the analysis gives the motion model and observation model needed by the SLAM algorithm for filtering prediction. The motion model is based on the model analysis of 
the IMU analyzed in this article, and the motion process equation is obtained through the accelerometer and gyroscope of the IMU; the observation model is based on the binocular stereo vision model analyzed in this article, and more accurate spatial coordinate points are obtained through binocular stereo matching. In view of the shortcomings of the FastSLAM algorithm, this paper introduces the immune algorithm and the particle swarm algorithm to solve the problem of particle degradation and particle exhaustion in the particle filtering process of the FastSLAM algorithm and improves the diversity of the sampled particles. Realizing the full intelligence of mobile robots is an ideal that people dream of. It is still impossible to develop a fully autonomous mobile robot in an unknown or complex changing environment, but as the technology matures, humans will intervene at a relatively low level, liberation from it, only high-level intervention.

\section{Conclusions}

This paper studies the intelligent robot control based on multi-sensor information fusion, focusing on the preprocessing of environmental information data collected by each sensor of the robot, processing and fusing the data collected by each sensor and planning the robot's movement path. In this paper, the famous sensor information fusion system is applied to outdoor mobile robot positioning, and the specific positioning filter design is researched and explored. Some phased results have been obtained, but there is still a long way to maturity and perfection. Outdoor mobile robots work in a real-time and dynamic environment, so the stability and reliability of the filter are highly demanded. In the simulation experiment, it is found that the performance of the filter is not stable, and sometimes it causes divergence due to bad data. The processing of bad data and the improvement in reliability need to be further studied and improved. Aiming at the problem of path planning in an unknown and static environment, a path planning method for mobile robots based on quantum genetic algorithm is proposed. Using a matrix encoding method in the form of random search, and introducing a reward function to optimize the fitness function, this method can better plan a collision-free feasible path for moving people in a known static environment. Simulation shows that the algorithm is effective and fast. Convergence speed, its convergence is better than standard genetic algorithm and quantum computing.

Acknowledgements

This work was supported by Talent Introduction Start-up Foundation (YJ2021-48), Middle-Young Aged Teachers'Technology Talent Development Foundation (ZQ2020-1), Shanghai Chenguang Program (20CG66), Shanghai Sailing Program (21YF1446600).

Authors' contributions

Yuan Guo and Xiaoyan Fang contributed to editing; Zhenbiao Dong and Honglin Mi contributed to data analysis. All authors read and approved the final manuscript.

Availability of data and materials

Data sharing does not apply to this article because no data set was generated or analyzed during the current research period.

\section{Declarations}

Ethics approval and consent to participate

This article is ethical, and this research has been agreed.

Consent for publication

The picture materials quoted in this article have no copyright requirements, and the source has been indicated. 
Competing interests

The authors declare that they have no competing interests.

\section{Author details}

${ }^{1}$ School of Mechanical Engineering, Shanghai Institute of Technology, Shanghai 201418, China. ${ }^{2}$ School of Foreign Languages, Shanghai Institute of Technology, Shanghai 201418, China.

Received: 4 August 2021 Accepted: 20 October 2021

Published online: 12 November 2021

\section{References}

1. B. Ji, M. Tao, H. Li, Research on simulation experiment of underwater cluster multi-source information fusion. IOP Conf Ser Earth Environ Sci 769(3), 032009 (2021)

2. W. Yi, M. Jiang, R. Hoseinnezhad et al., Distributed multi-sensor fusion using generalised multi-Bernoulli densities. IET Radar Sonar Navigat 11(3), 434-443 (2017)

3. D. Omid, T. Mojtaba, C.V. Raghvendar, IMU-based gait recognition using convolutional neural networks and multisensor fusion. Sensors 17(12), 2735 (2017)

4. G. Qin, S. Li, G. Xu, Research progress on multiscale entropy algorithm and its application in neural signal analysis. Sheng wu yi xue gong cheng xue za zhi J Biomed Eng Shengwu yixue gongchengxue zazhi 37(3), 541-548 (2020)

5. L. Huang, X. Yuan, J. Zhang et al., Research on internet of things technology and its application in building smart communities. J Phys Conf Ser 1550(2), 022029 (2020)

6. S. Yu, S. Nuske, S. Scherer, A multi-sensor fusion MAV state estimation from long-range stere. o, IMU GPS and barometric sensors 17(12), 11 (2017)

7. B. Xin, Y. Kawahara, Y. Wang et al., Efficient generalized fused lasso and its applications. ACM Trans Intell Syst Technol 7(4), 1-22 (2016)

8. J. Yu, D. Wei, G. Yao et al., Design of intelligent integrated power management system based on multi source information fusion. J Comput Theor Nanosci 14(3), 1473-1477 (2017)

9. H. Gang, L. Bing, Intelligent optimization of solar air heating system in large scale construction of and its application. Int J Smart Home 10(8), 99-106 (2016)

10. B. Koodziejska, N. Stpień, J. Kolmas, The influence of strontium on bone tissue metabolism and its application in osteoporosis treatment. Int J Mol Sci 22(12), 6564 (2021)

11. Z. Zhang, X. Xiong, Research on the method of new generation of multi-sensor information fusion technology to promote the development of wisdom agriculture in "internet +" mode. Fresen Environ Bull 25(12), 8030-8040 (2017)

12. H. Gao, J. Liu, X. Shen et al., Optimal power flow research in active distribution network and its application examples. Proc CSEE 37(6), 1634-1644 (2017)

13. G.D. Batur, S. Erol, O.E. Karasan, Robot move sequence determining and multiple part-type scheduling in hybrid flexible flow shop robotic cells. Comput Ind Eng 100, 72-87 (2016)

14. M. Sarkar, S. Dey, T. Kumar, Multi-objective neutrosophic optimization technique and its application to structural design. Int J Comput Appl 148(12), 31-37 (2017)

15. M.J. Al-Dujaili, A.Q. Zalzala, Implementation of SAR radar imaging algorithm on GPR and correction of its phase error in war mine detection. Sens Imaging 22(1), 1-17 (2021)

16. G. Sun, X. Guan, X. Yi et al., Numerical extending method for HFSs based on normative operator and its application in multi-attribute decision making. Xi Tong Gong Cheng Yu Dian Zi Ji Shu/Syst Eng Electron 40(7), 1530-1538 (2018)

17. P. Perdikaris, M. Raissi, A. Damianou et al., Nonlinear information fusion algorithms for data-efficient multi-fidelity modelling. Proc Math Phys Eng Sci 473(2198), 20160751 (2017)

18. A. Dameshghi, M.H. Refan, Wind turbine gearbox condition monitoring and fault diagnosis based on multi-sensor information fusion of SCADA and DSER-PSO-WRVM method. Int J Model Simul 39(1), 48-72 (2019)

19. G. Lei, Q. Lin, E. Chen et al., Discriminative multiple canonical correlation analysis for information fusion. IEEE Trans Image Process 99, 1-1 (2017)

20. L. Zhang, X. Wu, H. Zhu et al., Perceiving safety risk of buildings adjacent to tunneling excavation: an information fusion approach. Autom Constr 73, 88-101 (2016)

21. J. Guo, G. Hug, O.K. Tonguz, Intelligent partitioning in distributed optimization of electric power systems. IEEE Trans Smart Grid 7(3), 1249-1258 (2016)

22. El. Khoukhi, A. Fatima, Intelligent agents for dynamic optimization of learner performances in an online system. J Inf Technol Educ Res 16(1), 31-45 (2016)

23. C.Q. Ye, G.T. Ma, K. Liu et al., Intelligent optimization of an HTS Maglev system with translational symmetry. IEEE Trans Appl Supercond 26(4), 1-5 (2016)

24. Z. Lv, L. Qiao, K. Cai, Q. Wang, Big data analysis technology for electric vehicle networks in smart cities. IEEE Trans Intell Transp Syst 6, 66 (2020)

25. Z. LV, L. Qiao, Deep belief network and linear perceptron based cognitive computing for collaborative robots. Appl Soft Comput 6, 66 (2020)

\section{Publisher's Note}

Springer Nature remains neutral with regard to jurisdictional claims in published maps and institutional affliations. 